\title{
Facing Current Quantification Challenges in Protein Microarrays
}

\author{
Robert Wellhausen ${ }^{1,2,3}$ and Harald Seitz ${ }^{1,2}$ \\ ${ }^{1}$ Fraunhofer Institute for Biomedical Engineering (IBMT), Am Mühlenberg 13, 14476 Potsdam-Golm, Germany \\ ${ }^{2}$ Department of Vertebrate Genomics, Max-Planck Institute for Molecular Genetics, Ihnestrasse 73, 14195 Berlin, Germany \\ ${ }^{3}$ Institute for Chemistry and Biochemistry, Free University Berlin, Thielallee 63, 14195 Berlin, Germany \\ Correspondence should be addressed to Harald Seitz, harald.seitz@ibmt.fraunhofer.de
}

Received 10 November 2011; Revised 7 February 2012; Accepted 8 February 2012

Academic Editor: Zhirong Sun

Copyright ( $) 2012$ R. Wellhausen and H. Seitz. This is an open access article distributed under the Creative Commons Attribution License, which permits unrestricted use, distribution, and reproduction in any medium, provided the original work is properly cited.

The proteome is highly variable and differs from cell to cell. The reasons are posttranslational modifications, splice variants, and polymorphisms. Techniques like next-generation sequencing can only give an inadequate picture of the protein status of a cell. Protein microarrays are able to track these changes on the level they occur: the proteomic level. Therefore, protein microarrays are powerful tools for relative protein quantification, to unveil new interaction partners and to track posttranslational modifications. This papers gives an overview on current protein microarray techniques and discusses recent advances in relative protein quantification.

\section{Introduction}

As early as 1998, Büssow and coworkers [1] established a technique to catalogue protein products of arrayed cDNA clones. Closely after this successful effort to plot proteins on a macroarray, Lueking and colleagues [2] were able to produce protein microarrays from liquid expression cultures. They used a transfer stamp mounted to a spotting robot, which allowed the production of microarrays, setting a mark for high-throughput ligand receptor interaction studies. Since that time contact spotting for protein microarrays has mostly been replaced by piezoelectric, contact-free, spotting. For quantitative studies, it is mandatory that an equal amount of volume is transferred each time a sample is spotted on an array. Only this way, a highly reproducible array can be produced and the generated data quantified. This criterion is currently met only by piezoelectric spotting $[3,4]$.

In contrast to genomics, proteomics faces the fact that the proteome differs from organism to organism, between different tissues, and even between cells. Posttranslational modifications, splice variants, and polymorphisms are leading to a proteome that is temporally and spatially highly variable and differs from cell to cell. Different time points, for example, due to different states in the cell cycle or upon external stimulus, lead to a different protein composition of the cell [5]. Expression analysis of cells and tissues gives only an inadequate picture of the protein status in a cell. In contrast to that, protein microarrays are able to track these changes on the level they occur: the proteomic level. Before an external stimulus leads to an altered transcription profile and is manifested in a different proteome, the signal is passed through the cell by a consecutive set of posttranslational modifications of proteins. While analyzing signal transduction pathways, the problem comes up that only a subfraction of the whole proteome is of special interest. The proteins of high interest are kinases, phosphatases, receptors, ion channels, and transcription factors which are often low abundant proteins within the cell [6]. Therefore, the relative quantification of protein modifications is an important issue. However, most cell lysis methods fail to extract proteins from all cell compartments equally, and only a subfraction of this lysate is spotted on arrays. Thus the immobilized samples on slides represent only a small percentage of the whole proteome. As a direct consequence, detection mechanisms for the majority of proteins need to be very sensitive and accurate.

\section{Different Formats of Microarrays}

The term microarray is a collective term for a modern day technique used in research and development (R\&D) 




Figure 1: Modified from Hultschig et al. 2006 [7]. Different types of protein microarrays with their different substrates and detection methods. (a) After immobilization and (b) after incubation with different substrates.

as well as in in vitro diagnostics (ivD). Microarrays can be used to address different questions. Applications include DNA, RNA, protein, lysate, and peptide arrays. Therefore, they are able to cover proteomics and transcriptomics as well as genomics. DNA microarrays can analyze the whole transcriptome of a cell, represented by over one million DNA probes, whereas protein microarrays are mainly limited by the number of proteins. All microarrays offer the possibility for miniaturization and parallelization. This way precious sample material can be saved. Figure 1 depicts an overview on different microarray applications (a) and detection methods (b), which will be discussed in the following sections.

2.1. Antibody/Aptamer Arrays. Antibody microarrays and protein microarrays are often described as forward microarrays. The forward-phase or normal-phase protein microarray approach consists of the immobilization of a capture molecule (e.g., antibodies or aptamers, also known as prey) to a surface. The array is incubated with purified proteins, antibodies, or cell extract and detected as bait. This can be done either with directly labelled proteins or, in case of an immobilized prey antibody, with a second antibody that recognizes the bait (sandwich assay). Aptamers belong to the family of nucleic acids. Due to their 3D structure, they are a prominent compound used for target immobilization on microarray surfaces. Aptamers are used as affinity reagents in biosensor applications, because they show less crossreactivity than antibodies do [8]. Antibody microarrays have a broad field of application; for instances, we mention the following. (i) Detection of toxins [9] in spiked milk, apple cider, and blood samples; the detection limit was as low as $10-100 \mathrm{pg} / \mathrm{mL}$, which shows the high sensitivity of microarrays.

(ii) The progression of metastatic tissue can be detected with different markers [10]. Biomarkers are an important field in cancer research. With the help of antibody microarray experiments, up- and downregulation of several biomarkers involved in metastatic progression could be observed. All experiments conducted in this study showed correlation between protein microarray data and western blot experiments.

(iii) Detection of bacteria [11] was achieved by immobilizing IgG onto gold nanoparticle probes, capturing bacteria. Using antibody microarrays, discrimination between different bacterial strains could be done with as little as $10^{3}$ cells per assay. In addition to that, the capture could be inhibited by lipopolysaccharides, allowing the detection of minimum inhibitory concentrations, which are a valuable clinical parameter.

2.2. Peptide Arrays. Peptide microarrays have the advantage of being more stable than protein microarrays. The content can be adapted to any scientific question. Additionally they can be easily prepared and handled.

Kinase assays are a typical application for peptide microarrays, in order to screen for possible phosphorylation sites [12]. Furthermore, they are often used for serum profiling of diseases [13]. Peptides can be synthesized directly on the surface $[14,15]$, or the peptides are covalently 
immobilized on modified glass slides [16]. To identify target sites for kinases, the arrays are incubated either with cell lysate or recombinant kinases. During the incubation step radioactive labelled ATP is incorporated and can be detected afterwards [17]. Allen and coworkers [18] were the first ones describing an alternative detection method to radioactive labelled ATP with a similar sensitivity. Once a protein is phosphorylated with this ATP derivate, it can be transferred into a thiophosphate ester, which can be detected with a specific antibody. One of the advantages is the amplification step that comes with the use of a secondary antibody, while radioactive labelled ATP has no amplification.

However, the use of peptide microarrays is not limited to screen for kinase targets and the identification of potential phosphorylation sites or other posttranslational modifications like monoaminylation, methylation, or glycosylation. Excerpts from the fields of application include the following.

(i) The use of microarrays to identify peptides with immunogenic potential [19]; by 2010 , only $7 \%$ out of 4000 open reading frames of $M$. tuberculosis have been screened for T-cell epitopes. With a highthroughput screening method like peptide microarrays, 7446 overlapping peptides from 61 individual M. tuberculosis proteins were synthesized via the SPOT technique [20] and used to identify new potential T-cell epitopes.

(ii) The investigation of complex samples [21] using a peptide library which especially targeted cysteine proteases; cysteine proteases are involved in some pathological conditions. A binding study conducted with this library helped to find not only specific therapeutic inhibitors but also diagnostic markers. When tested against apoptotic Hela and red blood cells infected with Plasmodium falciparum, a specific and reproducible binding pattern can be observed. The obtained binding profiles could furthermore be linked to various stages of the parasite infection.

(iii) The use, to monitor enzymatic activity of proteins, for example, caspase activities; peptide microarrays were used for the detection of on chip enzymatic reactions, using the surface plasmon resonance technique. This way, lysates can be analyzed for their caspase activity, which provides valuable information for cell-based drug screening [22].

(iv) Solution-phase coupling of biotinylated synthetic peptides to NeutrAvidin [23]. Antibodies are immobilized to a NeutrAvidin-coated surface. Afterwards they can be detected using fluorescence-labelled secondary antibodies. With this technique, Andresen and colleagues were able to detect low-picomolar concentrations of monoclonal antibodies. These antibodies have been diluted in human serum, which demonstrates the potential of peptide microarrays for serum screening.

2.3. Lysate Array. Reverse-phase protein microarrays (RPMAs), also called lysate arrays, are generated by immobilizing small amounts of whole cell lysate onto any protein-binding surface. In principle RPMAs are miniaturized Dot blots. They have the potential to analyze small-quantity samples, at several time points, with different experimental settings and additionally several technical replications at once [24]. Technologies like DNA and RNA microarrays provide important results on expression levels, but the direct consequences of these expressional changes can hardly be assessed. Furthermore, they are unable to detect posttranslational modifications, which may define the phenotype and state of a cell [5]. Consequently the whole repertoire of patients' proteins, which represent the state of individual diseased tissue cell population, can be analyzed [25]. Quantitative immunoblotting [26] and MS [27] were used to make changes in protein levels visible; thus these techniques require large amounts of cellular material [28], which is a major drawback for systems working with primary material and/or stem cells. Additionally these techniques are time consuming and expensive.

Using the reverse-phase approach, a lot of different questions can be addressed; for instance, we mention the following.

(i) The relative impact of miRNAs on target proteins and their associated role in cellular processes [29]; because of different regulatory effects, it is hard to predict the actual impact of miRNAs. Protein lysate arrays are a tool to systematically monitor the effect on transfected cells. High-throughput transfections in breast cancer cells showed that out of 319 miRNAs, 21 downregulated the estrogen receptor-a. The results found by protein arrays could be verified by western blot and qPCR.

(ii) The detection of tissue-based markers [30]; as stated by Romeo and coworkers, lysate arrays measure changes on the proteomic level. Like RT-PCR and immunocytochemistry, lysate arrays have to be verified and normalized, too.

(iii) Time-dependent signal transduction [31]; Dupuy and colleagues investigated time-dependent phosphorylation pattern in complex signal transduction pathways. The study correlated with western blot analysis of the same samples while displaying excellent intra- and interslide reproducibility. Looking at the phosphorylation pattern of the transcription factor CREB, RPMAs showed a positive signal after one minute of stimulation with forskolin. Taken the already known CREB activation pattern into account, it can be estimated that the catalytic subunit of PKA translocates into the nucleus in less than a minute (R. Wellhausen, unpublished data).

These examples not only show the huge potential of reversephase protein microarrays but also clarify that strict operation procedure and validation methods are mandatory when working with lysate arrays. In the following section, key aspects to reproducible, valid, and quantitative arrays will be discussed. 
Validation of antibodies for use in reverse-phase protein microarrays (RPMA)

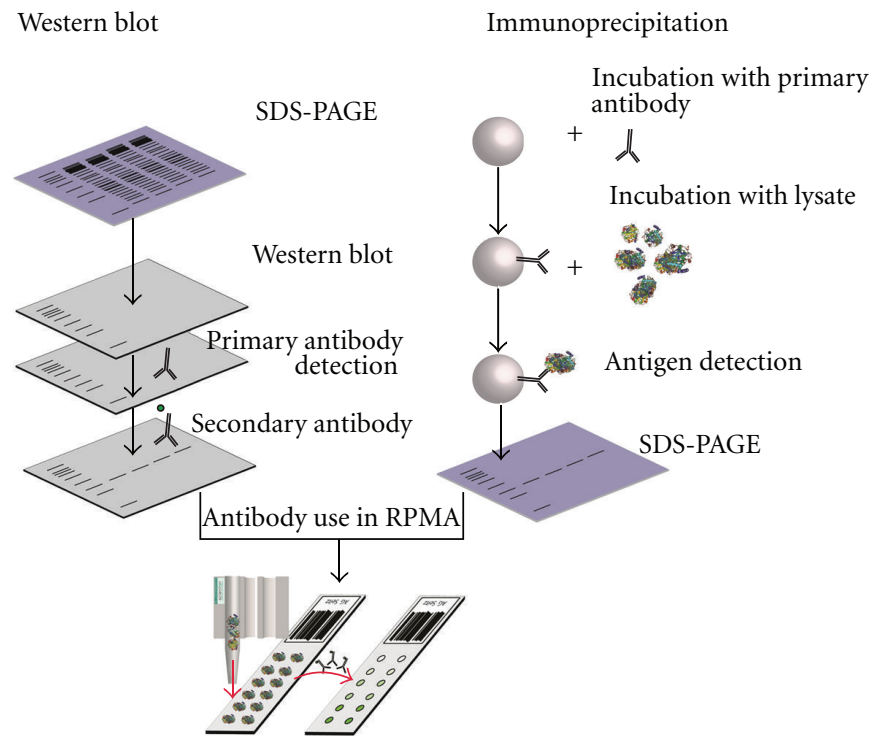

FIGURE 2: Strategy for antibody validation; only if single bands can be detected, the antibody can be used in microarray experiments.

\section{Validation of Antibodies/Detection}

There are different possibilities to detect the desired protein, depending on the array platform chosen. For any kind of protein microarray, the most common detection mechanisms are

(1) labelling of the cell extract,

(2) direct or indirect detection with labelled antibodies.

Labeling of proteins works only for antibody microarrays and has the disadvantage that it may mask the epitope; therefore the antigen cannot bind to the ligand on the surface [32]. Additionally a homogenous labelling of all proteins is a difficult task. Often high abundant proteins are preferably modified whereas low abundant proteins such as transcription factors are only rarely labelled.

Working with fluorescently labelled detection antibodies has the advantage of having a great dynamic range for quantification and signal amplification. Nevertheless, the antibodies should specifically detect their antigen and distinguish between closely related antigens. Lysate microarrays do not allow differentiating between homolog proteins nor do they separate proteins by their molecular mass. If the antibody itself has cross-reactivity or detects more than one antigen, the antibody cannot be used for a relative quantification. As a consequence the antibodies used in microarray experiments must be well characterized. Garcia and coworkers [33] showed that validation of antibodies is crucial. After validation, antibodies with a cross-reactivity below 5\% are selected, resulting in an analysis superior to traditional ELISA.

For a reliable antibody performance the results of Ambroz et al. [34] showed that detection reagents and conditions should be held constant for both antibody validation and microarray experiments. Different extraction reagents and methods lead to different states of a protein either it is in its native or in it is denaturated form [35]. To test if an antibody is capable of binding the linearized (denaturated) as well as the structured (native) form of the protein, all antibodies should undergo a validation. Ideally the antibody should yield a single band on a western blot (denatured condition) [36] and additionally should be able to bind to the native protein during immunoprecipitation experiments. One prominent strategy to test the specificity and selectivity is shown in Figure 2. If both, immunoprecipitation and western blot results are positive, the antibodies can be used in microarray experiments. The first test is western blot analysis. After SDS-PAGE, all proteins are denaturated; so, if a single band in a western blot can be detected, the antibody is capable of epitope recognition in their linearized (denaturated) form. The second test is an immunoprecipitation. In order to test whether the antibody binds to a native protein or not, the cells are lysed under native conditions. If the desired protein can be enriched via immunoprecipitation, the antibody is able to detect native epitopes and can, if both tests are passed positively, be used for readout and detection in microarray experiments.

\section{Blocking}

Proteins are either bound covalently to the surface (e.g., epoxy surfaces) or via unspecific adsorption (e.g., membrane). Taken the fact that it is not possible to cover the entire surface with the desired target protein, the detection antibody could bind unspecifically to the surface itself. This adsorption onto the surface leads to a higher background. Therefore the detection of a target protein is not only limited by its concentration but also by the background signals [37]. 


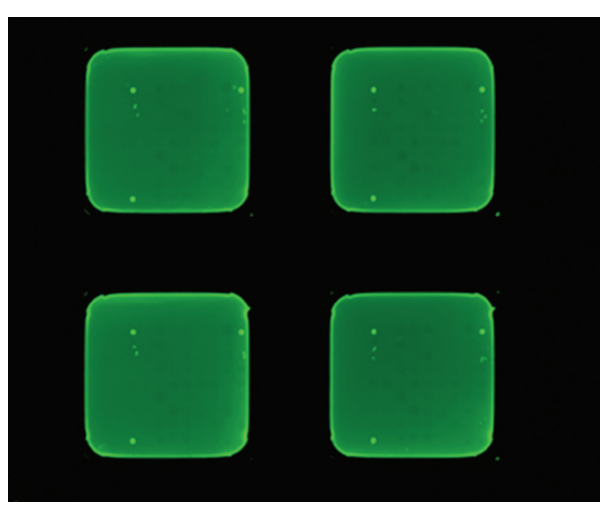

(a)

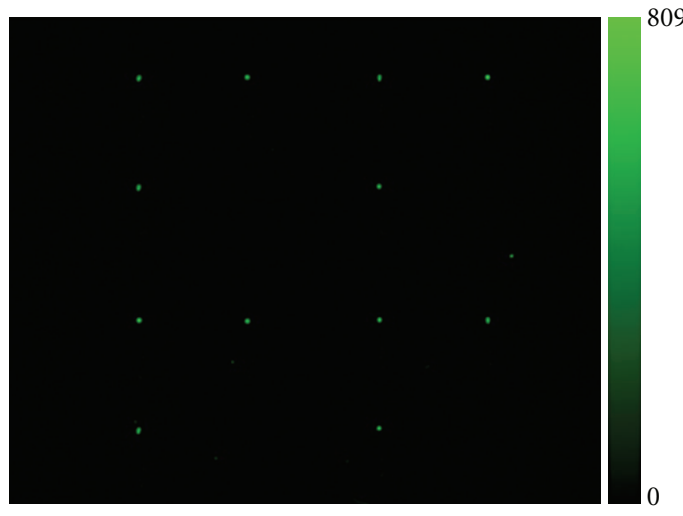

(b)

Figure 3: (a) Nitrocellulose- (b) epoxy-coated slides. Both show images of a RPPM after blocking with $10 \%$ skim milk for 1 hour at $4{ }^{\circ} \mathrm{C}$. (b) shows almost no background, whereas nitrocellulose (a) has a high background.

Different blocking buffers can have a significant effect on the intensity of the background, as well as changes in blocking conditions can influence antibody specificity [34]. To limit these influences blocking solutions containing nonfat/lowfat milk, bovine serum albumin, horse serum, or highly fluorinated organosilane are used to prevent nonspecific binding of analytes [38]. On an epoxy-coated slide 10\% skim milk powder appears to be the best blocking solution, resulting in the lowest background [39]. The direct comparison of a RPMA array with a nitrocellulose-coated slide and an epoxycoated slide after blocking is depicted in Figure 3. Both arrays were treated under the exact same conditions resulting in a higher background for the nitrocellulose slide (a) than for the epoxy-coated slide (b). When looking for low abundant proteins, a low background is crucial for the detection and analysis. The signal-to-noise ratio plays an important role in R\&D as well as in ivD, which will be discussed in the following section.

\section{Signal-to-Noise Ratio (SNR)}

The signal-to-noise ratio is used in many different applications. For microarrays, the SNR can help to distinguish whether the signal is a true signal or whether it is too weak to be considered for quantification. The SNR is used for all microarray applications to define positive signals. Only if the SNR is above a certain threshold, it is considered to be a true signal and can be used, for example, as a diagnostic marker. Achievement of a high signal-to-noise ratio gains even more importance when looking at low copy and abundant proteins. Regularly these proteins give only low-intensity signals. If the background is high, these signals might not be detected properly, or they might not be detected at all.

According to Rampal and coworkers [40], the signal-tonoise ratio is calculated as follows:

SNR

$=\frac{\text { average signal intensity }- \text { average background intensity }}{\text { standard deviations of background signals }}$.
SNR epoxy versus nitrocellulose

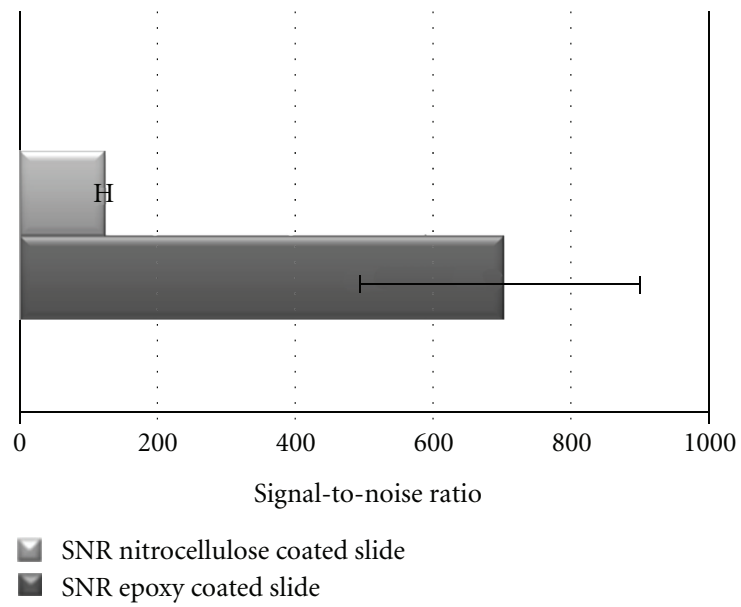

Figure 4: Comparison of the signal-to-noise ratio of the spots from Figure 3.

There is a direct correlation between the slide surface (i.e., the natural occurring material background intensity and the background intensity after blocking) and the SNR. As depicted in Figure 5 the signal-to-noise ratio is higher for epoxy-coated slides than for nitrocellulose-coated slides. The demand for a high SNR comes with quantification itself. When it comes to $\mathrm{R} \& \mathrm{D}$, sometimes a yes or no answer is needed, which can also be given by a weak signal. Decisions made with the help of in vitro diagnostics have to be as certain as possible, in order to eliminate the number of false positive or negative signals.

\section{Mass Transport}

After printing and blocking of a protein microarray, the target molecules need to be detected.

As depicted in Figure 4, the signal-to-noise ratio is higher with epoxy-coated slides compared to nitrocellulose-coated 
Mean versus median intensity

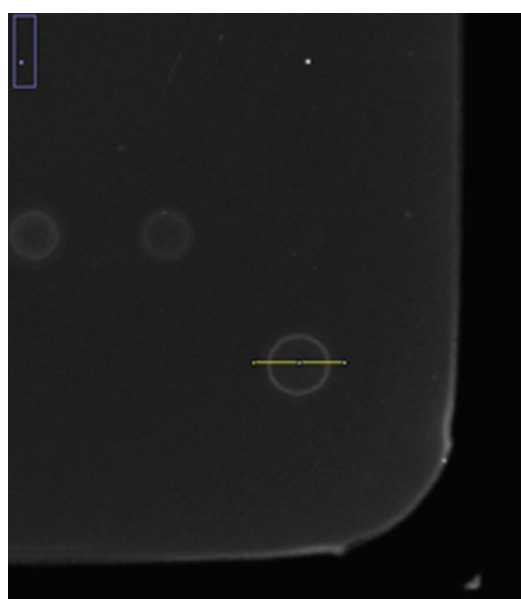

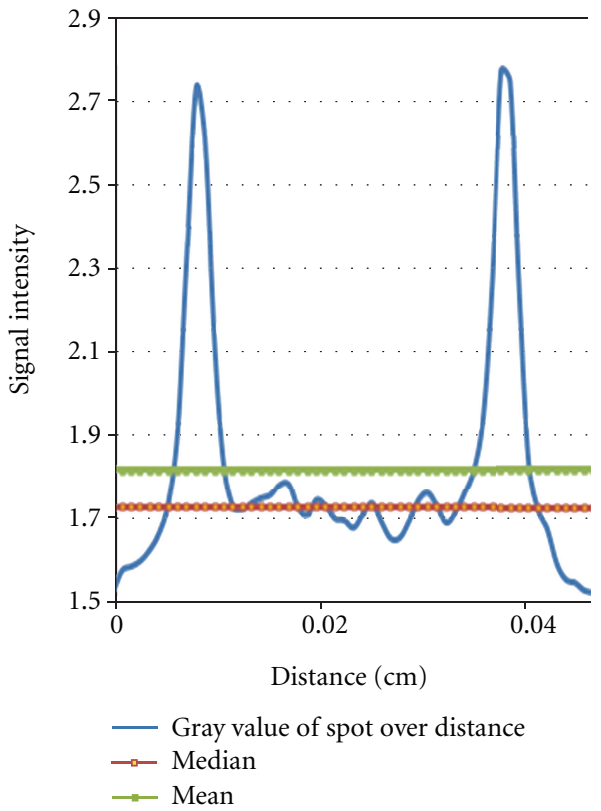

(b)

FIGURE 5: Mean versus median spot intensity for donut structures. Left-hand picture shows a protein spot with a typical donut structure (orange line indicates the intensity intersection). Right-hand side: the intensity distribution is plotted (red and green line are mean and median signal intensity of the spot).

slides. If the substrate allows immobilization on epoxycoated surfaces, it should be chosen over nitrocellulosecoated slides.

When incubating protein microarrays with specific antibodies, one limitation is the mass transport of the sample and detection antibodies to the surface. In microspot immunoassays, the binding event of an antibody to an antigen is slowed down by several orders of magnitude compared to well-stirred bulk reactions. Therefore several factors need to be taken under consideration: buffer viscosity, no mixing or imperfect mixing of the array, incubation time, analyte concentration, and the use of antibodies with higher affinities [41]. Sometimes it is not possible to take all these points under consideration, the buffer composition for certain extraction procedures cannot be changed, or there is no antibody available that has a higher affinity. Up to a certain level, it is possible to make up for these issues with elongated incubation times and improved mixing. To overcome the problems of mass transport, the mixing and washing volumes are crucial parameters. If working with a gasket/chamber system, the applicable volume is limited. Therefore the incubation and washing times have to be extended.

\section{Mean versus Median}

Protein spots often come with a ring-shaped structure, referred to as donut structure. During the formation of such a structure, the proteins are accumulating at the outer boundary of a spot. This particular structure makes it hard to analyze the signal, because the intensity is unevenly distributed throughout the spot. The intensity/pixel ratio is much higher on the outer rim than in the middle of the spot. To overcome this problem, spotting and drying conditions have to be optimized dependent on the immobilization chemistry, the buffer used, and several other factors. Humidity in the spotting chamber can be regulated, so that the spots will not dry immediately after being dispensed. However, these changes not guarantee to overcome the occurring problems of ring-shaped structures. During drying the proteins are accumulating preferably at the outer boundary of the spots. Zhu and Guo [42] state that protein molecules are known to preferentially accumulate at air/water interfaces. Detergents like Triton X-100 lower the surface tension of the spotting mix and can be used to reduce this effect.

However, if the above-mentioned methods fail and the donut-shaped structure persists, it can be mathematically adjusted. Measuring the mean and median spot intensities gives an idea of the actual value. If the gray value is distributed as shown in Figure 5, it makes more sense to take the median intensity, to really cover the majority of all values. If the spot has a homogeneous morphology, both values mean and median are almost the same. After that, it comes to a case-to-case decision where it has to be mathematically and statistically evaluated if the signal has to be excluded from the array.

\section{Normalization}

Normalization and standardization of samples, microarrays, and microarray data itself are of importance. As far as 
changes on the proteomic level are concerned, small changes of PTMs or the expression level itself matter. However, these small changes cannot be estimated if, for example, the different lysates on the arrays are not spotted under equimolar conditions. Therefore, the samples have to be quantified before spotting using techniques like BCA or Bradford. The total amount of bound protein can be determined after spotting and washing but before further processing like blocking. If the amount of total protein differs from spot to spot, the signal intensities can be normalized within one array and between different slides. This way, fold changes of proteins or their PTM status (via antibody detection) can be accurately detected and interpreted correctly.

\section{Conclusion}

Protein microarrays are a powerful tool not only to identify, for example, new interaction partners or posttranslational modifications, but also for relative protein quantification. They contribute greatly to the investigation and understanding of the proteome on a quantitative level. During the last years, a lot of work and effort has been put into optimizing protocols and standard operation procedures. These procedures should be carried out at all times when working with protein microarrays. All antibodies used in array experiments have to be controlled beforehand, to make sure that the detected signals are valid. Commercially available antibodies are marked for their intended use, for example, recommended for use in immunohistochemistry, western blot analysis, or immunoprecipitation. The same kind of information should be provided for the use of antibodies in microarray experiments. The slides' surface has to be chosen carefully in order to find a suitable platform for each individual purpose. Blocking buffers have to be optimized each time the surface changes and should be kept constant for both the validation of antibodies and the array experiments itself. Incubation should be carried out under conditions limiting mass transport problems. The SNR should be critically observed at all times to be sure that the signal can be quantified. The individual structure for each spot has to be determined in order to find a suitable mathematical model for analysis. If all these points are taken care of, protein microarrays will greatly contribute to help understanding proteomic procedures and will play an important role in clinical diagnostics as well as in research and development.

\section{Acknowledgments}

The authors would like to thank Prof. Dr. F. Herberg and colleagues for the assistance with kinase arrays, M. Alhamdani, and K. Köhler for helpful discussions. This work was supported by the German Ministry for Research (BMBF Grant 0315449D).

\section{References}

[1] K. Büssow, D. Cahill, W. Nietfeld et al., "A method for global protein expression and antibody screening on high-density filters of an arrayed cDNA library," Nucleic Acids Research, vol. 26, no. 21, pp. 5007-5008, 1998.

[2] A. Lueking, M. Horn, H. Eickhoff, K. Büssow, H. Lehrach, and G. Walter, "Protein microarrays for gene expression and antibody screening," Analytical Biochemistry, vol. 270, no. 1, pp. 103-111, 1999.

[3] E. T. Fung and J. B. Delehanty, "Printing functional protein microarrays using piezoelectric capillaries," in Protein Arrays, pp. 135-143, Humana Press, 2004.

[4] C. Löbke, M. Laible, C. Rappl et al., "Contact spotting of protein microarrays coupled with spike-in of normalizer protein permits time-resolved analysis of ERBB receptor signaling," Proteomics, vol. 8, no. 8, pp. 1586-1594, 2008.

[5] R. Tibes, Y. H. Qiu, Y. Lu et al., "Reverse phase protein array: validation of a novel proteomic technology and utility for analysis of primary leukemia specimens and hematopoietic stem cells," Molecular Cancer Therapeutics, vol. 5, no. 10, pp. 2512-2521, 2006.

[6] G. L. G. Miklos and R. Maleszka, "Protein functions and biological contexts," Proteomics, vol. 1, no. 2, pp. 169-178, 2001.

[7] C. Hultschig, J. Kreutzberger, H. Seitz, Z. Konthur, K. Büssow, and H. Lehrach, "Recent advances of protein microarrays," Current Opinion in Chemical Biology, vol. 10, no. 1, pp. 4-10, 2006.

[8] J. R. Collett, J. C. Eun, and A. D. Ellington, "Production and processing of aptamer microarrays," Methods, vol. 37, no. 1, pp. 4-15, 2005.

[9] W. Lian, D. Wu, D. V. Lim, and S. Jin, "Sensitive detection of multiplex toxins using antibody microarray," Analytical Biochemistry, vol. 401, no. 2, pp. 271-279, 2010.

[10] W. Shi, Z. Meng, Z. Chen, J. Luo, and L. Liu, "Proteome analysis of human pancreatic cancer cell lines with highly liver metastatic potential by antibody microarray," Molecular and Cellular Biochemistry, vol. 347, no. 1, pp. 117-125, 2011.

[11] J. Gao, C. Liu, D. Liu, Z. Wang, and S. Dong, "Antibody microarray-based strategies for detection of bacteria by lectinconjugated gold nanoparticle probes," Talanta, vol. 81, no. 4-5, pp. 1816-1820, 2010.

[12] M. Uttamchandani et al., "A peptide microarray for detecting protein kinase activity in cell lysates," in Small Molecule Microarrays, pp. 183-194, Humana Press, 2010.

[13] W. Hueber, B. A. Kidd, B. H. Tomooka et al., "Antigen microarray profiling of autoantibodies in rheumatoid arthritis," Arthritis and Rheumatism, vol. 52, no. 9, pp. 2645-2655, 2005.

[14] S. Matysiak, F. Reuthner, and J. D. Hoheisel, "Automating parallel peptide synthesis for the production of PNA library arrays," BioTechniques, vol. 31, no. 4, pp. 896-904, 2001.

[15] R. Frank, "The SPOT-synthesis technique: synthetic peptide arrays on membrane supports-principles and applications," Journal of Immunological Methods, vol. 267, no. 1, pp. 13-26, 2002.

[16] N. Kimura, T. Okegawa, K. Yamazaki, and K. Matsuoka, "Sitespecific, covalent attachment of poly(dT)-modified peptides to solid surfaces for microarrays," Bioconjugate Chemistry, vol. 18, no. 6, pp. 1778-1785, 2007.

[17] C. J. Hastie, H. J. McLauchlan, and P. Cohen, "Assay of protein kinases using radiolabeled ATP: a protocol," Nature Protocols, vol. 1, no. 2, pp. 968-971, 2006.

[18] J. J. Allen, M. Li, C. S. Brinkworth et al., "A semisynthetic epitope for kinase substrates," Nature Methods, vol. 4, no. 6, pp. 511-516, 2007.

[19] S. Gaseitsiwe, D. Valentini, S. Mahdavifar, M. Reilly, A. Ehrnst, and M. Maeurer, "Peptide microarray-based identification 
of Mycobacterium tuberculosis epitope binding to HLADRB1*0101, DRB1*1501, and DRB1*0401," Clinical and Vaccine Immunology, vol. 17, no. 1, pp. 168-175, 2010.

[20] B. Spurrier, F. L. Washburn, S. Asin, S. Ramalingam, and S. Nishizuka, "Antibody screening database for protein kinetic modeling," Proteomics, vol. 7, no. 18, pp. 3259-3263, 2007.

[21] H. Wu, J. Ge, P.-Y. Yang, J. Wang, M. Uttamchandani, and S. Q. Yao, "A peptide aldehyde microarray for high-throughput profiling of cellular events," Journal of the American Chemical Society, vol. 133, no. 6, pp. 1946-1954, 2011.

[22] Y. Inoue, T. Mori, G. Yamanouchi et al., "Surface plasmon resonance imaging measurements of caspase reactions on peptide microarrays," Analytical Biochemistry, vol. 375, no. 1, pp. 147-149, 2008.

[23] H. Andresen, C. Grötzinger, K. Zarse, O. J. Kreuzer, E. Ehrentreich-Förster, and F. F. Bier, "Functional peptide microarrays for specific and sensitive antibody diagnostics," Proteomics, vol. 6, no. 5, pp. 1376-1384, 2006.

[24] S. M. Chan, J. Ermann, L. Su, C. G. Fathman, and P. J. Utz, "Protein microarrays for multiplex analysis of signal transduction pathways," Nature Medicine, vol. 10, no. 12, pp. 1390-1396, 2004.

[25] C. P. Pjaweletz, L. Charboneau, V. E. Bichsel et al., "Reverse phase protein microarrays which capture disease progression show activation of pro-survival pathways at the cancer invasion front," Oncogene, vol. 20, no. 16, pp. 1981-1989, 2001.

[26] I. Swameye, T. G. Müller, J. Timmer, O. Sandra, and U. Klingmüller, "Identification of nucleocytoplasmic cycling as a remote sensor in cellular signaling by databased modeling," Proceedings of the National Academy of Sciences of the United States of America, vol. 100, no. 3, pp. 1028-1033, 2003.

[27] Y. Zhang, A. Wolf-Yadlin, P. L. Ross et al., "Time-resolved mass spectrometry of tyrosine phosphorylation sites in the epidermal growth factor receptor signaling network reveals dynamic modules," Molecular and Cellular Proteomics, vol. 4, no. 9, pp. 1240-1250, 2005.

[28] U. Korf, S. Derdak, A. Tresch et al., "Quantitative protein microarrays for time-resolved measurements of protein phosphorylation," Proteomics, vol. 8, no. 21, pp. 4603-4612, 2008.

[29] S. K. Leivonen, R. Mäkelä, P. Östling et al., "Protein lysate microarray analysis to identify microRNAs regulating estrogen receptor signaling in breast cancer cell lines," Oncogene, vol. 28, no. 44, pp. 3926-3936, 2009.

[30] M. J. Romeo, J. Wunderlich, L. Ngo, S. A. Rosenberg, S. M. Steinberg, and D. M. Berman, "Measuring tissue-based biomarkers by immunochromatography coupled with reverse-phase lysate microarray," Clinical Cancer Research, vol. 12, no. 8, pp. 2463-2467, 2006.

[31] L. Dupuy, C. Gauthier, G. Durand et al., "A highly sensitive near-infrared fluorescent detection method to analyze signalling pathways by reverse-phase protein array," Proteomics, vol. 9, no. 24, pp. 5446-5454, 2009.

[32] G. MacBeath, "Protein microarrays and proteomics," Nature Genetics, vol. 32, no. 5, pp. 526-532, 2002.

[33] B. H. Garcia II, A. Hargrave, A. Morgan et al., "Antibody microarray analysis of inflammatory mediator release by human leukemia T-cells and human non small cell lung cancer cells," Journal of Biomolecular Techniques, vol. 18, no. 4, pp. 245-251, 2007.

[34] K. L. H. Ambroz, Y. Zhang, A. Schutz-Geschwender, and D. M. Olive, "Blocking and detection chemistries affect antibody performance on reverse phase protein arrays," Proteomics, vol. 8, no. 12, pp. 2379-2383, 2008.
[35] V. Espina, E. C. Woodhouse, J. Wulfkuhle, H. D. Asmussen, E. F. Petricoin, and L. A. Liotta, "Protein microarray detection strategies: focus on direct detection technologies," Journal of Immunological Methods, vol. 290, no. 1-2, pp. 121-133, 2004.

[36] S. M. Major, S. Nishizuka, D. Morita et al., "AbMiner: a bioinformatic resource on available monoclonal antibodies and corresponding gene identifiers for genomic, proteomic, and immunologic studies," BMC Bioinformatics, vol. 7, article 192, 2006.

[37] B. B. Haab, M. J. Dunham, and P. O. Brown, "Protein microarrays for highly parallel detection and quantitation of specific proteins and antibodies in complex solutions," Genome Biology, vol. 2, no. 2, Article ID RESEARCH0004, 2001.

[38] H. Y. Hsieh, P. C. Wang, C. L. Wu, C. W. Huang, C. C. Chieng, and F. G. Tseng, "Effective enhancement of fluorescence detection efficiency in protein microarray assays: application of a highly fluorinated organosilane as the blocking agent on the background surface by a facile vapor-phase deposition process," Analytical Chemistry, vol. 81, no. 19, pp. 7908-7916, 2009.

[39] M. S. S. Alhamdani, C. Schröder, and J. D. Hoheisel, "Analysis conditions for proteomic profiling of mammalian tissue and cell extracts with antibody microarrays," Proteomics, vol. 10, no. 17, pp. 3203-3207, 2010.

[40] J. B. Rampal et al., "Forward-phase and reverse-phase protein microarray," in Microarrays, pp. 363-373, Humana Press, 2007.

[41] W. Kusnezow, Y. V. Syagailo, S. Rüffer et al., "Kinetics of antigen binding to antibody microspots: strong limitation by mass transport to the surface," Proteomics, vol. 6, no. 3, pp. 794-803, 2006.

[42] X. Zhu and A. Guo, "The critical role of surface chemistry in protein microarrays," in Functional Protein Microarrays in Drug Discovery, pp. 53-71, CRC Press, 2007. 

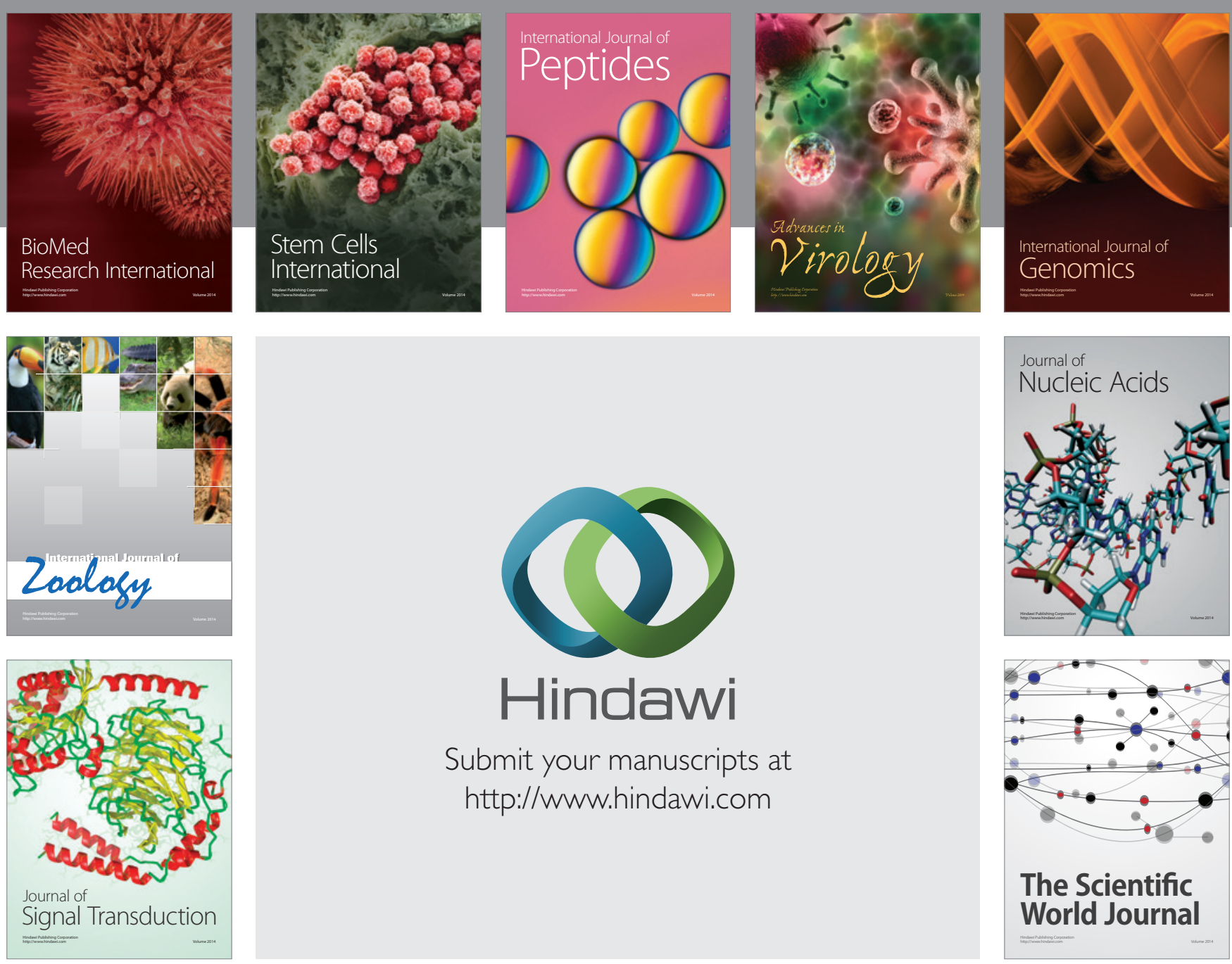

Submit your manuscripts at

http://www.hindawi.com
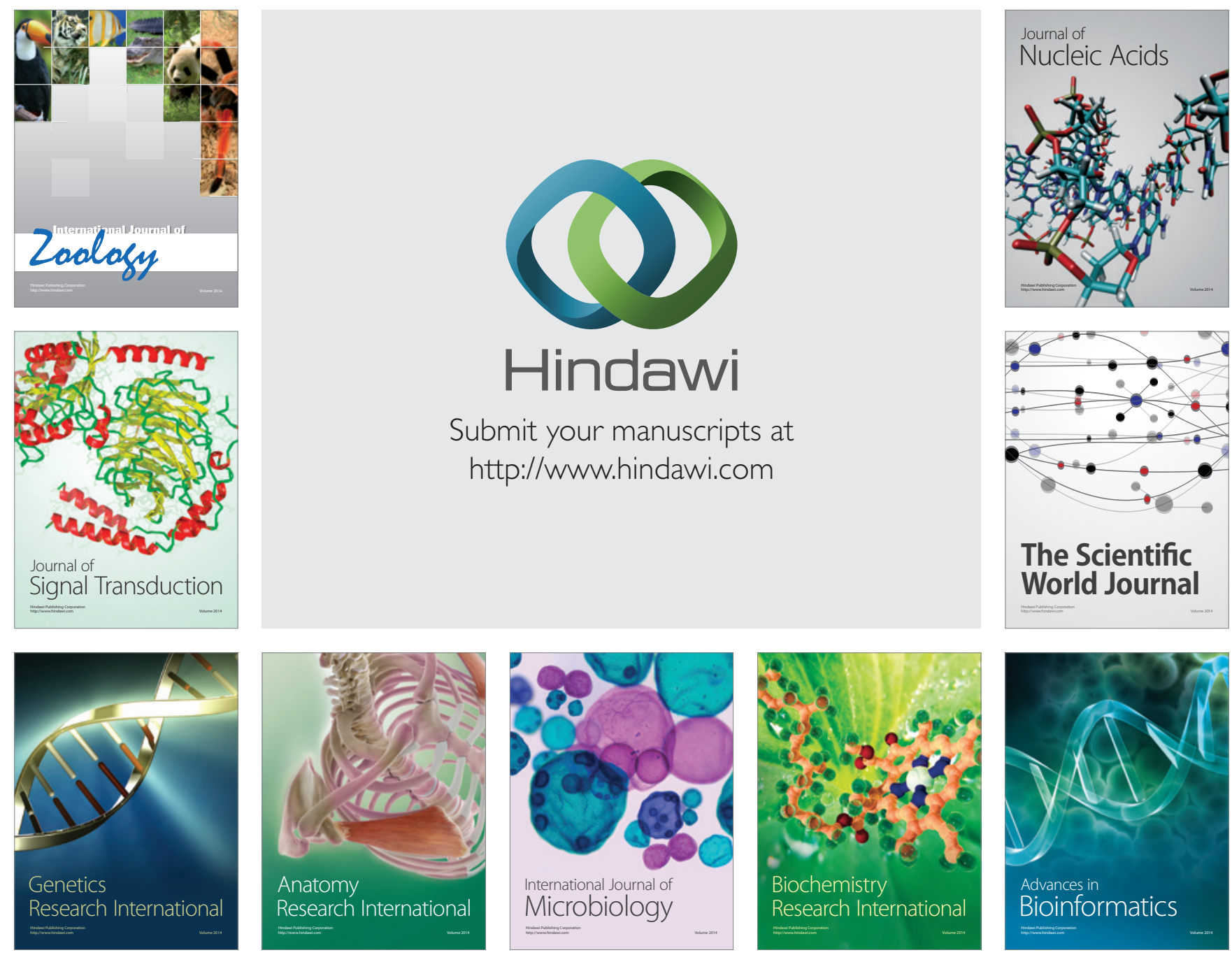

The Scientific World Journal
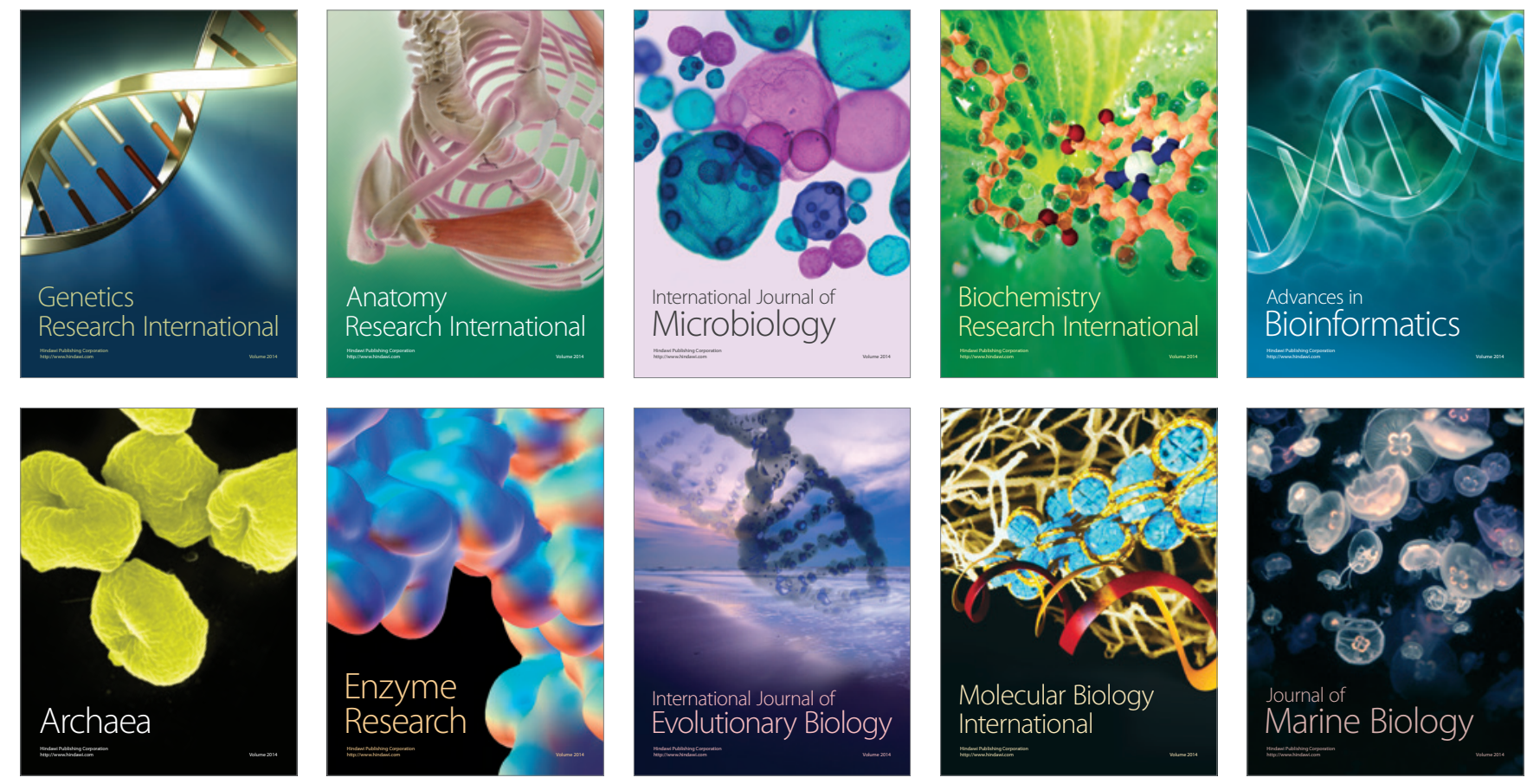